\title{
Modifying risk factors: do the means justify the ends?
}

\author{
Stephen B Hanauer
}

Maybe I like to use clichés, but I do think we are losing our ability to see the forest for the trees. We are quite adept at identifying risk factors for common diseases, but our approach to modifying these factors often seems backwards or, at least, reactive rather than proactive. This hasn't always been the case. Indeed, on a personal note, back in 1972 I took my fiancée to an allergist to treat asthma that had been induced by exposure to dog hair. He refused to treat her unless she rid herself of the dog (which was mine), recognizing that this was a totally preventable condition and knowing that the pharmacological therapies available had inherent risks and costs.

Today, it is ironic that, as a society, the US is relying on drug therapies to modify risk factors for diseases that are directly related to common and preventable health issues. For example, with the childhood obesity epidemic leading to greater risks of developing heart disease, there have been proposals to initiate statin therapy in children who have increased cholesterol levels. At the same time physical education has virtually disappeared from US public schools (at least in large cities) as a (short-term) supposedly cost-saving measure, and unhealthy, carbohydrate-ridden and fatridden meals are being provided to pupils. Why are we considering the administration of statins to try to modify heart disease that might develop 40 years hence and not promoting healthy lifestyles, foods and exercise?

In their Perspective on "Redefining Quality Implications of Recent Clinical Trials" Krumholz and Lee discuss different paradigms aimed at disease prevention focusing on modification of known risk factors (New Engl J Med [2008] 358: 2537-2539). They point out that “...the

\section{In medicine, the means \\ to achieve \\ surrogate \\ end-points \\ can often \\ really impact \\ the ultimate \\ intended \\ outcomes}

SB Hanauer is Editorin-Chief of Nature Clinical Practice Gastroenterology \& Hepatology.

\section{Competing interests \\ The author declared no} competing interests.

www.nature.com/clinicalpractice doi:10.1038/ncpgasthep1229 way in which risk factors are modified really does matter" and discuss several clinical trials that attempted to gain tighter control of cholesterol and glucose levels. Despite success at achieving risk factor modification, the interventions used led to negative outcomes that outweighed the intended benefits. Krumholz and Lee then note that "Lifestyle interventions may have few risks, but we cannot assume the same for drugs - and drug related risks are not always known or appreciated." Indeed, it is probable that different strategies used to achieve the same treatment goal, particularly when there are surrogate risk factors for disease outcomes, make a difference-at least in certain subgroups.

Our Gl societies are emphasizing and appropriately supporting treatment of obesity - but the horse is already out of the barn. We've seen the negative impacts of pharmaceutical regimens for weight reduction (e.g. the development of valvular heart disease) and these must be the tip of the iceberg. Substituting drug therapy for lifestyle modifications of known risk factors cannot possibly be as safe or cost-effective.

As described by Krumholz and Lee, there are numerous examples of how different strategies used to achieve the same outcome will have different inherent risks and costs (particularly when the outcomes are risk factors rather than the ultimate disease end-points). It is, therefore, relevant to compare different strategies for achieving the same intended outcome. In medicine, the means to achieve surrogate end-points can often really impact the ultimate intended outcomes.

P.S. We found the dog a new home and have been married and asthma free for 36 years. 